\title{
Chest pain and non-respiratory symptoms in acute asthma
}

\author{
W M Edmondstone
}

\begin{abstract}
The frequency and characteristics of chest pain and non-respiratory symptoms were investigated in patients admitted with acute asthma. One hundred patients with a mean admission peak flow rate of $38 \%$ normal or predicted were interviewed using a questionnaire. Chest pain occurred in $76 \%$ and was characteristically a dull ache or sharp, stabbing pain in the sternal/parasternal or subcostal areas, worsened by coughing, deep inspiration, or movement and improved by sitting upright. It was rated at or greater than $5 / 10$ in severity by $67 \%$ of the patients. A wide variety of upper respiratory and systemic symptoms were described both before and during the attack.

Non-respiratory symptoms occur commonly in the prodrome before asthma attacks and become more frequent after onset of the attack. Chest pain is usual during asthma attacks. Although it is benign and self limiting it may cause diagnostic confusion and patient distress. (Postgrad Med F 2000;76:413-414)
\end{abstract}

Keywords: asthma; chest pain; non-respiratory symptoms

The Royal Hospital, Haslar, Gosport, Hants PO12 2AA, UK

Correspondence to: Dr Edmondstone

Submitted 26 October 1999 Accepted 20 December 1999

Characteristic symptoms of asthma are breathlessness, chest tightness, wheeze, and cough. Although physicians may be familiar with the chest pain described by some asthmatics during attacks the phenomenon has not been studied, nor does it merit a mention in most

Table 1 Chest symptoms described by 100 patients

\begin{tabular}{|c|c|c|c|}
\hline Breathlessness & \multicolumn{3}{|c|}{$=100 \%$} \\
\hline Wheezing & \multicolumn{3}{|c|}{$=94 \%$} \\
\hline Chest tightness & \multicolumn{3}{|c|}{$=91 \%$} \\
\hline Cough & \multicolumn{3}{|c|}{$=88 \%$} \\
\hline Chest pain & \multicolumn{3}{|c|}{$=76 \%$} \\
\hline \multicolumn{4}{|c|}{ Features of the chest pain (\% patients affected) } \\
\hline Location: & \multicolumn{2}{|c|}{ Quality: } & \\
\hline Sternal/parasternal & 30 & Dull ache & 30 \\
\hline Subcostal & 26 & Sharp and stabbing & 28 \\
\hline Chest and back & 26 & Dull ache + sharp and stabbing & 17 \\
\hline Sternal and subcostal & 9 & Others (burning, raw, abrasive, tight) & 25 \\
\hline Back only & 6 & & \\
\hline Others & 3 & & \\
\hline Time started: & & verity: (scale $1-10$ ) & \\
\hline With onset of attack & 37 & 9 & 5 \\
\hline Within $60 \mathrm{~min}$ of onset & 25 & 8 & 17 \\
\hline $1-6$ hours & 16 & 7 & 19 \\
\hline $6-24$ hours & 4 & 6 & 12 \\
\hline$>24$ hours & 18 & $<6$ & 47 \\
\hline Worsened by: & & proved by: & \\
\hline Coughing & 84 & Sitting upright & 66 \\
\hline Deep inspiration & 47 & Shallow breathing & 22 \\
\hline Movement & 43 & Keeping still & 18 \\
\hline Lying supine & 36 & Holding chest & 12 \\
\hline Lying on side & 12 & Lying on side & 9 \\
\hline PF manoeuvre & 8 & Lying supine & 8 \\
\hline Sitting up without support & 3 & Stretching & 7 \\
\hline
\end{tabular}

NB: Most patients reported several factors. textbooks. Occasionally the combination of dyspnoea and chest pain results in diagnostic confusion. This study was prompted by the observation that a number of patients admitted with asthmatic chest pain had been suspected of having cardiac ischaemia, pleurisy, pericarditis, or pulmonary embolism. It had also been observed that many patients admitted with asthma complained of a range of nonrespiratory symptoms, something which has been noted previously in children ${ }^{1}$ and in adult asthmatics in outpatients. ${ }^{2}$ The aim of this study was to examine the frequency and characteristics of chest pain and other symptoms in patients admitted with acute asthma.

\section{Patients and methods}

One hundred patients (66 females, mean (SD) age 45.0 (19.7) years) admitted with acute asthma were studied. Patients were included if a compatible history was supported by an admission peak expiratory flow (PEF) of less than $50 \%$ predicted or previous best. Patients readmitted were not studied a second time. The patients were interviewed within two days of admission using a questionnaire that asked about symptoms including the presence and features of chest pain, and new non-respiratory symptoms during the attack and the 24 hours preceding its onset. Patients reporting chest pain were interviewed again before discharge or by telephone soon after.

\section{Results}

The mean (SD) PEF on admission expressed as $\%$ predicted or previous best was 37.9 (12.1)\% and on discharge was 87.0 (13.7)\%. Seventy six patients reported chest pain during the attack of asthma. Details of the pain are in table 1. Five patients had two different pains such as retrosternal soreness due to tracheitis and a second pain elsewhere in the chest or back.

Typically the pain developed gradually over the first few hours of the attack, was located in the sternal/parasternal area, and was dull and aching or sharp and stabbing in nature. In some patients it was a dull ache that became sharp and stabbing on coughing, deep inspiration, or change in position. These activities also worsened the pain in $84 \%, 47 \%$, and $43 \%$ of patients respectively. The pain was often relieved by sitting upright. Of those who had chest pain its severity was rated at or above $7 / 10$ in $41 \%$, and although only $22 \%$ received analgesics, many more would have liked them. All the patients reported that their pain eased as the asthma attack settled, and 69 (91\%) patients were pain free when they were discharged. Of the others, in five the pain was 
Table 2 Non-respiratory symptoms associated with the asthma attack (\% patients)

\begin{tabular}{lll}
\hline Symptoms & $\begin{array}{l}\text { Before the } \\
\text { attack }\end{array}$ & $\begin{array}{l}\text { During the } \\
\text { attack }\end{array}$ \\
\hline Nose and throat symptoms & 50 & 54 \\
Tiredness/sleepiness & 48 & 52 \\
Dry mouth & 43 & 66 \\
Thirst & 42 & 59 \\
Sweating & 32 & 61 \\
Headache & 32 & 43 \\
Depression & 28 & 28 \\
Skin flush & 21 & 37 \\
Increased urinary frequency & 21 & 30 \\
Irritability & 21 & 29 \\
Palpitations & 19 & 41 \\
Skin itch & 17 & 20 \\
Limb pains & 15 & 16 \\
Nausea & 11 & 30 \\
Dizziness & 9 & 36 \\
Faintness & 9 & 23 \\
Limb tingling & 9 & 19 \\
Abdominal colic & 7 & 14 \\
Weight change & 7 & - \\
\hline
\end{tabular}

thought to be due to rib fracture or intercostal muscle tear. In only one case did an area of consolidation on the chest radiograph correspond to the site of the pain, and in no other case did the radiograph help to explain the pain. There were no differences in the severity of the asthma attack between those who did, and those who did not have chest pain. Four of the patients with chest pain had radioisotope lung scans. All were normal. Electrocardiograms were done in $36 \%$ of those with chest pain and were normal apart from showing sinus tachycardia.

The commonest non-respiratory symptoms during the 24 hours before the asthma attack were irritation of the nose and throat, frequently coryzal in nature, but other common symptoms including excessive tiredness or sleepiness, dry mouth, thirst, sweating, and headache also occurred (table 2). After the start of the attack the frequency of nonrespiratory symptoms increased with the exception of depression.

\section{Discussion}

This study has demonstrated that chest pain is common in acute severe asthma. In some patients tracheitis may precede or accompany the attack, while in others repeated violent coughing may provoke tears where the intercostal muscles insert into the periosteum of the rib, or even rib fracture. Occasionally the pain may be pleuritic due to pneumonia. In most cases in this study, however, features of the pain suggest that it is musculoskeletal in origin: its temporal relationship to the asthma attack, its localisation around the costochondral joints and lower ribs, and its relationship to position and movement of the thorax. The chest hyperinflates during severe asthma and ventilation occurs at lung volumes approaching total lung capacity. This increases the work of breathing as the respiratory muscles are required to operate at a mechanical disadvantage, resulting in muscle fatigue and consequent pain. Altered

\section{Learning points}

- Most patients with acute asthma have chest pain that may cause diagnostic confusion and be severe enough to require analgesia, but which settles as the asthma improves.

- Many asthmatics develop prodromal symptoms before attacks and may be able to recognise impending attacks.

thoracic geometry may also result in painful distortion of the costochondral joints.

Surprisingly, asthmatic chest pain has been neglected in adults, although paediatricians are aware of its importance in the differential diagnosis of exertional chest pain. ${ }^{3}$ Its significance should not be underestimated. Firstly, it can lead to diagnostic uncertainty, evidenced in this study by unnecessary radioisotope lung scans in some patients. Secondly, many patients are apprehensive about the pain and may need reassurance that it is a predictable and self limiting feature of acute asthma, and finally they may need analgesia.

This study has also shown that nonrespiratory symptoms frequently occur both before and during attacks of asthma. Many of the prodromal symptoms are explained by coryzal upper respiratory inflammation, and many of the symptoms during the attack are probably due to anxiety, lack of sleep, repeated coughing, side effects of bronchodilators, and mouth breathing due to nasal obstruction. Hyperventilation may account for the limb tingling, dizziness, and faintness. None the less, some patients reported prodromal symptoms without having an upper respiratory infection, while others said that their asthma attacks were predictably preceded by harbinger symptoms such as tingling, itching, or scratchy discomfort in the nose, mouth, and throat. Mood change, including inappropriate depression or irritability, itching of the skin around the nose or mouth, and urinary frequency were commonly reported, and some patients noted muscular aches and pains in the absence of other features of influenza. Whether these symptoms, in some respects similar to the prodromal symptoms of migraine, are due to release of inflammatory mediators in advance of the asthma attack is not known. Patients whose prodromal symptoms follow a stereotypic pattern may be able to avert acute attacks by increasing the dose of their preventer medication when they recognise the characteristic features.

1 Beer S, Laver J, Karpuch J, et al. Prodromal features of asthma. Arch Dis Child 1987;62:345-8.

2 Shneerson J. Non-respiratory symptoms of acute asthma. Thorax 1986;41:701-2.

3 Wiens L, Sabath R, Ewing L, et al. Chest pain in otherwise Wiens L, Sabath R, Ewing L, et al. Chest pain in otherwise
healthy children and adolescents is frequently caused by exercise-induced asthma. Pediatrics 1992;90:350-3. 